\title{
Adaptive Mechanisms of Asphodelus aestivus Brot. to Withstand Drought Stress: Metabolic Constituents and Activity of Antioxidant Enzymes.
}

\author{
Amal A. Morsy", Raifa A. Hassanein*, Nahed M. Nour \\ El - Din ${ }^{* *}$, Aesha H. Abdel Kawy ${ }^{* * *}$ \\ *Botany Department, Faculty of Science, Ain Shams University \\ and ${ }^{* *}$ Plant Ecology and Range Management Department, \\ Desert Research Center, Cairo, Egypt.
}

\begin{abstract}
7 HE SEASONAL effects on the ecophysiological responses of Asphodelus aestivus Brot. (A. microcarpus) were investigated. Changes in water content, degree of succulence, osmotic potential, photosynthetic pigments, carbohydrate fractions, mineral contents, total lipids, total phospholipids and activities of certain antioxidant enzymes were investigated in leaves and root tubers of the studied plant. Soil moisture content decreased generally during autumn season. Total chlorophyll,carotenoid, $\mathrm{K}^{+}, \mathrm{Mg}^{+2}, \mathrm{P}^{+3}$ and total carbohydrate contents, in leaves in early autumn, showed an increase however root tubers exhibited an increase in most ions such as $\mathrm{K}^{+}, \mathrm{Na}^{+}, \mathrm{Ca}^{+2}, \mathrm{Cl}^{-}$and $\mathrm{P}^{+3}$. Asphodelus aestivus showed a specific defense strategy against ROS to achieve adaptation by increasing activities of specific enzymes such as superoxide dismutase (SOD), peroxidase (POD), catalase (CAT), Polyphenol oxidase (PPO) and GR glutathione reductase (GR), in root tubers, SOD, GR and Glutathione peroxidase (GPX) in leaves. Asphodelus aestivus has developed different defense strategies against drought stress and completed a specific life form cycle, which might be considered as an intermediate stage between avoidance and tolerance to being well adapted against water stress.
\end{abstract}

Keywords: Asphodelus aestivus, Antioxidant Enzymes, Oxidative Stress, Reactive Oxygen Species.

Asphodelus aestivus Brot. (A. microcarpus Viv.), family Asphodelaceae (Asparagales) (Boulos, 2005), is aperennial spring-flowering geophyte, widely distributed over the Mediterranean basin. Its formations represent the last degradation stage of the Mediterranean type ecosystems. These ecosystems often (referred to as "Asphodel deserts" or "Asphodel semideserts") result from drought, frequent fires, soil erosion and overgrazing. It is found in both in arid and semi-arid Mediterranean ecosystems and in certain regions of North Africa. Asphodelus aestivus, as other geophytes (cryptophytes), has a considerable distribution, since it has become the dominant life form in many degraded Mediterranean ecosystems. A. aestivus has two major phenological phases within a year.

An active one (autumn - late spring) from leaf emergence to the senescence of the above-ground structures (photosynthetic period) and an inactive (summer) phase (dormancy), which lasts until the emergence of leaves (Rhizopoulou et al., 
1997). A. aestivus is a sessile organism reproducing by means of root tubers as well as by seeds. These facts are of considerable importance regarding maintenance and even dominance of $A$. aestivus within degraded areas.

Shewry (2003) suggested two roles for tuberous roots: first, to store carbon and nitrogen in forms that can be mobilized when required; and second, to sprout and produce new plants as propagules. The underground tuber is rich in starch and anthraquinones and plays a role in traditional medicine and pharmacological action and toxicity. As far as pharmacological actions, it can be used as a laxative and a purgative. It is used in folk and traditional medicine and treats the digestive system and skin ailments. In some countries, it is used externally to treat ulcers and its seeds are used to make a kind of dried milk. The ability of $A$. aestivus, a native floristic element, to spread and to dominate in all areas over the Mediterranean region reflects its capacity to face not only the peculiarities of the Mediterranean climate, but also to resist the most common disturbances in its habitat. The present study aimed to understand the adaptive mechanisms of $A$. aestivus through investigating the metabolic changes and antioxidant defense system that are related to the two phases in life form of the studied plant and concomitantly, to climatic periodicity (autumn and winter).

\section{Materials and Methods}

\section{Geomorphology of the studied area}

the studied area of Wadi El- Maktala of Sedi Barrani located between latitude $31^{\circ} 33^{\prime}$ North and longitude $26^{\circ} 10^{\prime}$ East. It lies $140 \mathrm{~km}$ after Matrouh and $90 \mathrm{~km}$ before El-Sallum in the North Western Coast. Geomorphologically, Sedi Barrani area is located near the Mediterranean Sea about $95 \mathrm{~km}$ east of the border with Libya, and about $420 \mathrm{~km}$ west of Alexandria (Fig. 1). Maktala sector comprises a vast area of fertile land (about 80 square $\mathrm{km}$ ) with a more or less relatively dense cover of natural vegetation (Migahid et al., 1974). The completely western desert (including the studied area) is one of the extremely arid parts of the world.

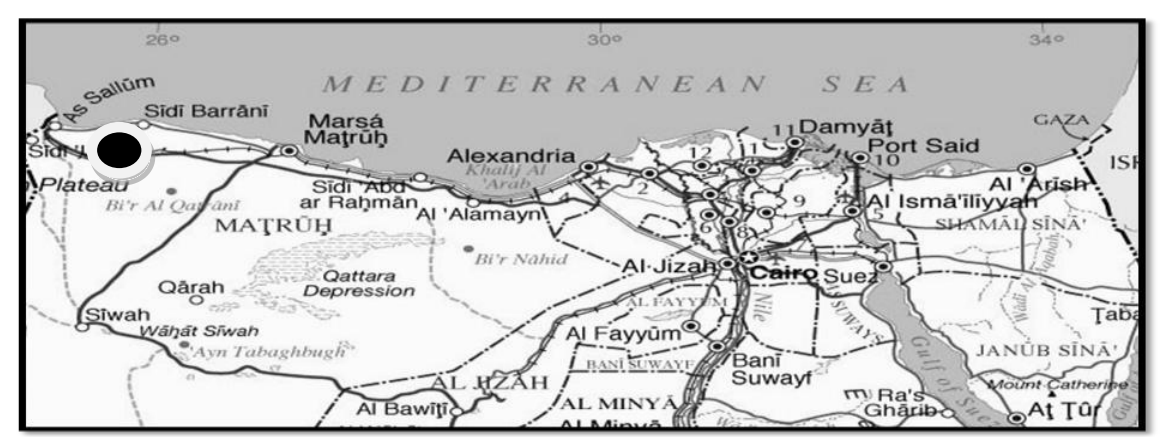

Fig. 1. Location map showing the studied site of sedi barrani of the western coastal region of Egypt.

Egypt. J. Bot., Vol. 56, No. 1 (2016) 


\section{Climate}

the climatic data of the studied area was collected from the meteorological station of El Sallum, which is the nearest of Sedi Barrani. Maximum and minimum mean values of temperature $\left({ }^{\circ} \mathrm{C}\right)$, the relative humidity $(\%)$, rainfall $(\mathrm{mm} / \mathrm{months})$ and wind velocity $(\mathrm{km} / \mathrm{h})$ were recorded for the selected site.

\section{Soil analysis}

samples of soil associating the plant were collected from a depth of 0-20 and $20-40 \mathrm{~cm}$. Physical and chemical parameters of such soil samples were analyzed and presented in Table 1. Soil samples were air - dried and sieved through $2 \mathrm{~mm}$ sieve for mechanical analysis. Soil water extract was prepared according to Rowell (1994). Electrical conductivity of the soil extract was expressed as mmhos/ $\mathrm{cm}$ and the soil reaction $(\mathrm{pH})$ was measured by means of a glass electrode $\mathrm{pH}$ meter according to Rowell (1994). Chlorides were estimated by $\mathrm{AgNO}_{3}$ titration method according to Jackson (1967).

The organic matter content, bicarbonates, carbonates, sulphate concentrations were determined by the chemical methods described by Rowell (1994). Sodium and potassium were determined using flame photometer according to Rowell (1994), whereas calcium and magnesium were evaluated by the versinate titration methods according to Harris (1998). Phosphorus was determined colorimetrically according to the method of Chapman and Pratt (1961). Nitrogen was estimated using micro-Kjeldahel method as described by James, (1995). Microelements such as $\mathrm{Fe}^{+3}, \mathrm{Mn}^{+2}, \mathrm{Zn}^{+2}$ and $\mathrm{Cu}^{+2}$ were determined using Atomic Absorption Spectrophotometer (Buck Scientific Model 210VGP AAS, East Norwalk, USA) according to the method described by Harris (1998). The results of cations and anions were expressed as meq/L.

\section{Plant materials}

samples of leaves and root tubers of Asphodelus aestivus were collected for ecophysiological and biochemical studies during one - year period (August 2011 to February - 2012), from its natural habitat. Sampling was conducted in a $10 \times 10 \mathrm{~m}$ area from 3 different plants on each sampling date, and all the experiments were repeated at least 3 times. After the tuberous roots were dissected from the plants, they were washed with tap water, then with distilled water and were kept in icebox.

\section{Eco-physiological analysis}

changes in water content, degree of succulence, osmotic potential, photosynthetic pigments, carbohydrate fractions, mineral contents, total lipids, phospholipids and activities of antioxidant enzymes; superoxide dismutase (SOD), catalase (CAT), ascorbate peroxidase (APX), glutathione reductase (GR), Polyphenol oxidase (PPO) and peroxidase (POD) were examined and compared during two seasons for leaveand tuberof the studied plant. Glutathione peroxidase (GPX) activity was also determined in the leaves.Water content was analyzed according to the method recommended by Rowell, (1994). 
Degree of succulence was calculated according to initial fresh weight / dry weight ratio as described by Hoolbrook and Putz (1996). According to Simmelsgaard, (1976), the sap was used directly for osmotic potential determination in vapor pressure Osmometer. The photosynthetic pigments (Chlorophyll a, Chlorophyll b and carotenoids) were determined for mature leaves by a spectrophotometric method as recommended by Metzner et al. (1965). The concentration of total soluble sugars, polysaccharides and total carbohydrates were determined colorimetrically according to Homme et al. (1992) and Smith et al., (1964). Plant materials were also digested according to the method of Rowell (1994).

The acid digest of the plant materials was analyzed for the determination of minerals. Sodium and potassium were determined using the flame photometer according to Rowell (1994), whereas calcium and magnesium were evaluated by the versinate titration methods according to Harris (1998). Phosphorus was determined colorimetrically according to the method of Chapman and Pratt (1961). Sulphate concentrations were determined by the turbidmetric method as described by Rowell (1994). Chlorides were estimated by the silver nitrate titration method according to Jackson and Thomas (1960).

The percentage of total lipids was determined according to AOAC (2000), while phospholipids were determined by colorimetric method according to Connerty et al., (1961). The assay of POD (EC 1.11.1.7) was carreid out according to Hammerschmidt et al. (1982), CAT (EC 1.11.1.6) activity was determined by the method of Goel et al. (2003). The activity of SOD (EC 1.15.1.1) was measured according to Misra and Fridovich (1972), PPO (EC 1.10.3.1) activity was determined according to Mayer et al. (1965) and APX (EC.1.11.1.11) activity was measured according to Nakano and Asada (1981). For GR (EC.1.6.4.2) activity, Foyer and Halliwell (1976) method was used and for GPX (EC.1.11.1.9) activity, the method of Wendel (1981) was adopted.

\section{Statistical analysis}

data were statistically analyzed to determine the effect of season on each parameter using one way complete randomized design .Test of significance was done according to the least significant difference (LSD) at 5\% level of probability. Values presented are mean \pm standard deviation of three replicates. Data of ecophysiological parameters were analyzed by using COSTAT software programme, update version.

\section{Results}

\section{Climate:}

according to the meteorological data obtained for the studied area from 2004 -2013 , it appears that the climate of the region belongs to the warm coastal desert. The meteorological data show that, summer months (July and August) are the hottest months, while those of the winter (December, January and February) are the coldest ones.

Egypt. J. Bot., Vol. 56, No. 1 (2016) 


\section{Soil analysis}

the texture of the soil associated with Asphodelus aestivus was mainly of loamy sand during both autumn and winter seasons (Table 1).

TABLE 1. Granulometric analysis of the soil associated with Asphodelus aestivus plant at Sedi Brrani.

\begin{tabular}{|c|c|c|c|c|c|c|c|c|c|c|c|}
\hline \multirow[b]{2}{*}{$\begin{array}{c}\text { Soil } \\
\text { depth } \\
\text { cm }\end{array}$} & \multirow[b]{2}{*}{$\begin{array}{l}\text { Season of } \\
\text { sampling }\end{array}$} & \multicolumn{7}{|c|}{ Granulometric analysis } & \multirow[b]{2}{*}{$\begin{array}{c}\text { Soil } \\
\text { Texture }\end{array}$} & \multirow[b]{2}{*}{$\begin{array}{c}\text { Soil } \\
\text { moisture } \\
\%\end{array}$} & \multirow[b]{2}{*}{$\begin{array}{c}\text { Saturation } \\
\text { point } \\
\%\end{array}$} \\
\hline & & $\begin{array}{c}\text { Gravel } \\
2> \\
\mathrm{Mm}\end{array}$ & $\begin{array}{c}\text { Very } \\
\text { Coarse } \\
\text { sand } \\
2-1 \\
\text { Mm }\end{array}$ & $\begin{array}{c}\text { Coarse } \\
\text { sand } \\
1-0.5 \\
\text { Mm }\end{array}$ & $\begin{array}{c}\text { Medium } \\
\text { sand } \\
0.5-0.25 \\
\text { Mm }\end{array}$ & $\begin{array}{l}\text { Fine } \\
\text { sand } \\
0.25- \\
\mathbf{0 . 1 2 5} \\
\mathrm{Mm}\end{array}$ & $\begin{array}{c}\text { Very } \\
\text { Fine } \\
\text { sand } \\
\mathbf{0 . 1 2 5 -} \\
\mathbf{0 . 0 6 3} \\
\text { Mm }\end{array}$ & $\begin{array}{c}\text { Silt } \\
\text { \&Clay } \\
<0.063 \\
\text { Mm }\end{array}$ & & & \\
\hline \multirow{2}{*}{$0-20$} & Autumn & 0.75 & 1.00 & 2.34 & 29.26 & 31.09 & 19.87 & 15.69 & Sandy & 0.34 & 28 \\
\hline & Winter & 10.46 & 2.04 & 6.30 & 28.61 & 23.74 & 17.56 & 11.29 & Sandy & 3.26 & 28 \\
\hline \multirow{2}{*}{$20-40$} & Autumn & 2.16 & 1.43 & 3.25 & 31.82 & 27.91 & 17.92 & 15.51 & Sandy & 1.40 & 27 \\
\hline & Winter & 2.58 & 4.35 & 11.03 & 33.00 & 21.85 & 16.80 & 10.39 & Sandy & 3.69 & 28 \\
\hline
\end{tabular}

The soil moisture content attained higher values in winter while it reached a value of $3.69 \%$ at the depth $20-40 \mathrm{~cm}$ and tend to increase as depth increased. The saturation percentage was almost the same in autumn and winter. The electrical conductivity (EC) of the soil extract ranged from 0.40 to 0.77 mmhos /cm during autumn and 0.67 to $0.77 \mathrm{mmhos} / \mathrm{cm}$ during winter (Table 2). Soil reaction was alkaline (7.91during autumn and 7.98 during winter) at the surface layer. However, at the deeper layer, soil reaction was slightly alkaline ranged from 7.79 during autumn to 7.63 during winter.

\section{Cation content}

it is clear from Table 2 that $\mathrm{Na}^{+}$increased during winter compared with autumn at the surface layer while the opposite trend occurred at the deeper layer, mean while $\mathrm{K}^{+}$increased during winter at both depths compared with autumn. On the other hand, $\mathrm{Ca}^{+2}$ increased during autumn compared with the rainy one at the surface layer while, it gives the same value at the deeper layer. Furthermore, $\mathrm{Mg}^{+2}$ attained higher values at the surface layer compared with those recorded at 20-40 cm depth.

\section{Anion content}

Table 2 shows that the soluble carbonates were present as traces in the both depths, giving negligible values except during season at depth of $20-40 \mathrm{~cm}$. The amount of bicarbonates was high $(4.00 \mathrm{meq} / \mathrm{L})$ in winter especially at the depth of $0-20 \mathrm{~cm}$. Chlorides were generally high during autumn and being $5.50 \mathrm{meq} /$ $\mathrm{L}$ at the surface layer and $6.10 \mathrm{meq} / \mathrm{L}$ at the deeper one. Sulphates showed the same trend where they decreased during winter and this reduction was more obvious at the depth of $0-20 \mathrm{~cm}$. Data in Table 2 indicate that the concentrations of nitrogen and phosphorous were relatively high during autumn. 
TABLE 2. Physical and chemical analysis of the soil associated with Asphodelus aestivus plant at Sedi Brrani.

\begin{tabular}{|c|c|c|c|c|c|c|c|c|c|}
\hline \multirow{2}{*}{$\begin{array}{c}\text { Soil } \\
\text { depth } \\
\text { cm }\end{array}$} & \multirow{2}{*}{ Se. } & \multirow{2}{*}{$\begin{array}{c}\text { E.C } \\
\text { mmhos } \\
\text { /cm }\end{array}$} & \multirow{2}{*}{ pH } & \multirow{2}{*}{$\begin{array}{c}\text { O.C } \\
\%\end{array}$} & \multirow{2}{*}{$\begin{array}{c}\text { T.S.S } \\
\%\end{array}$} & \multicolumn{4}{|c|}{ Cations $(\mathrm{meq} / \mathrm{L})$} \\
\hline & & & & & & $\mathbf{N a}$ & $\mathbf{K}$ & $\mathbf{C a}$ & Mg \\
\hline \multirow{2}{*}{$0-20$} & A. & 0.40 & 7.91 & 4.20 & 0.03 & 1.45 & 1.51 & 8.00 & 6.00 \\
\hline & W. & 0.67 & $\begin{array}{l}7.98 \\
\end{array}$ & 3.70 & 0.05 & 1.50 & 1.05 & 7.00 & 9.80 \\
\hline \multirow{2}{*}{$20-40$} & A. & 0.77 & 7.79 & 3.20 & 0.05 & 1.45 & 1.76 & 8.60 & 1.40 \\
\hline & W. & 0.77 & 7.63 & 4.20 & 0.05 & 1.40 & 1.20 & 8.60 & 1.40 \\
\hline
\end{tabular}

Se. :Season, A: Autumn, W: Winter

TABLE 2 Cont.

\begin{tabular}{|c|c|c|c|c|c|c|c|c|c|c|c|}
\hline \multirow{2}{*}{$\begin{array}{c}\text { Total } \\
\text { soluble } \\
\text { cations } \\
\text { meq/L }\end{array}$} & \multicolumn{4}{|c|}{ Anions (meq/L) } & \multirow{2}{*}{$\begin{array}{c}\text { Total } \\
\text { soluble } \\
\text { anions } \\
\text { meq/L } \\
\end{array}$} & \multirow{2}{*}{$\begin{array}{c}\mathrm{N} \\
\mathrm{meq} / \mathrm{L}\end{array}$} & \multirow{2}{*}{ P meq/L } & \multicolumn{4}{|c|}{ Microelements (meq/L) } \\
\hline & $\mathrm{HCO}_{3}$ & $\mathrm{CO}_{3}$ & $\mathrm{Cl}$ & $\mathrm{SO}_{4}$ & & & & $\mathbf{F e}$ & Mn & $\mathbf{Z n}$ & $\mathrm{Cu}$ \\
\hline 16.96 & 2.00 & Nil & 5.50 & 0.90 & 8.40 & 0.52 & 0.11 & 0.14 & 0.012 & 0.001 & $<0.006$ \\
\hline 19.35 & 4.00 & Nil & 5.00 & 0.44 & 9.44 & 0.15 & 0.09 & 0.002 & 0.001 & 0.001 & $<0.006$ \\
\hline 13.21 & 2.00 & 0.20 & 6.10 & 0.56 & 8.86 & 0.52 & 0.11 & 0.07 & 0.010 & 0.003 & $<0.006$ \\
\hline 12.60 & 2.00 & Nil & 6.00 & 0.31 & 8.31 & 0.15 & 0.11 & 0.002 & 0.001 & 0.003 & $<0.006$ \\
\hline
\end{tabular}

There was no difference between the two depths, where it varied from 0.52 and $0.11 \mathrm{meq} / \mathrm{L}$ in autumn to 0.15 and 0.09 or $0.11 \mathrm{meq} / \mathrm{L}$ during winter respectively. $\mathrm{Fe}^{+3}$ and $\mathrm{Mn}^{+2}$ were higher during autumn at both depths whereas, $\mathrm{Zn}^{+2}$ showed the opposite trend and $\mathrm{Cu}^{+2}$ had no difference during both seasons at the two depths.

Eco-physiological studies in root tubers

No significant differences were observed in water content during the two seasons Table 3 . Tubers were attained the lowest value $(59.9 \pm 0.60 \%)$ of water content during autumn compared to that recorded during winter $(79.7 \pm 3.83)$. On the other hand, results recorded in Table 3 show that the difference in the degree of succulence, similar to that of water content where the highest value

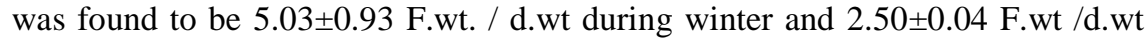
during autumn.

As for carbohydrates, polysaccharides and total carbohydrates were decreased during autumn $(3.51 \pm 2.21,14.1 \pm 1.66 \mathrm{~g} \%)$ compared to winter $(13.0 \pm 0.87,23.7 \pm 0.88 \mathrm{~g} \%)$ respectively. Total lipids tend to increase from $5.47 \pm 0.06$ during autumn to $8.57 \pm 0.15 \mathrm{~g} / 100 \mathrm{~g}$ during winter. Opposite trend in mineral contents and enzyme activities were observed in Table 3 where the highest values of $\mathrm{Na}^{+}, \mathrm{K}^{+}, \mathrm{Ca}^{+2}, \mathrm{Cl}^{-}, \mathrm{P}^{+3}$ were recorded during autumn except for $\mathrm{K}^{+} / \mathrm{Na}^{+}$ratio, $\mathrm{Mg}^{+2}, \mathrm{SO}_{4}^{-2}$ and APX which showed marked decrease. 
ADAPTIVE MECHANISMS OF ASPHODELUS AESTIVUS BROT...

TABLE 3. Seasonal variations of some metabolic status and antioxidant enzymes ( $\mathrm{U} \mathrm{g}^{-1} \mathrm{~F}$. wt) of Asphodelus aestivus root tubers.

\begin{tabular}{|c|c|c|c|c|c|c|}
\hline \multirow{2}{*}{\multicolumn{3}{|c|}{ Parameters }} & \multirow{3}{*}{$\begin{array}{l}\text { Seasons } \\
\text { Autumn } \\
\end{array}$} & \multicolumn{3}{|c|}{ Root tubers } \\
\hline & & & & Mean \pm & L.S.D & Sig. \\
\hline \multirow{4}{*}{ Water relations } & \multirow{2}{*}{\multicolumn{2}{|c|}{ Water content $(\%)$}} & & $59.9 \pm 0.60$ & \multirow{2}{*}{6.21} & \multirow{2}{*}{$* *$} \\
\hline & & & Winter & $79.7 \pm 3.83$ & & \\
\hline & \multirow{2}{*}{\multicolumn{2}{|c|}{$\begin{array}{l}\text { Degree of succulence } \\
\text { (fresh wt / dry wt ) }\end{array}$}} & Autumn & $2.50 \pm 0.04$ & \multirow{2}{*}{1.49} & \multirow{2}{*}{ ** } \\
\hline & & & Winter & $5.03 \pm 0.93$ & & \\
\hline \multirow{6}{*}{ Sugars $(\mathrm{g} \%)$} & \multirow{2}{*}{\multicolumn{2}{|c|}{ Total soluble sugars }} & Autumn & $10.6 \pm 0.56$ & \multirow{2}{*}{----} & \multirow{2}{*}{$\mathrm{n}$} \\
\hline & & & Winter & $10.7 \pm 0.65$ & & \\
\hline & \multirow{2}{*}{\multicolumn{2}{|c|}{ Polysaccharides }} & Autumn & $3.51 \pm 2.21$ & \multirow{2}{*}{3.81} & \multirow{2}{*}{$* *$} \\
\hline & & & Winter & $13.0 \pm 0.87$ & & \\
\hline & \multirow{2}{*}{\multicolumn{2}{|c|}{ Total carbohydrates }} & Autumn & $14.1 \pm 1.66$ & \multirow{2}{*}{3.01} & \multirow{2}{*}{$* *$} \\
\hline & & & Winter & $23.7 \pm 0.88$ & & \\
\hline \multirow{2}{*}{ lipids } & \multirow{2}{*}{\multicolumn{2}{|c|}{ Total lipids ( g / 100 g dry wt) }} & Autumn & $5.47 \pm 0.06$ & \multirow{2}{*}{0.26} & \multirow{2}{*}{ ** } \\
\hline & & & Winter & $8.57 \pm 0.15$ & & \\
\hline \multirow{16}{*}{ Minerals } & & $\mathrm{Na}^{+}$ & Autumn & $20.0 \pm 0.87$ & 255 & ** \\
\hline & & $\mathrm{Na}$ & Winter & $7.54 \pm 1.33$ & 2.55 & wa \\
\hline & & $\mathrm{K}^{+}$ & Autumn & $40.0 \pm 2.05$ & & ${ }^{*}$ \\
\hline & & $\mathrm{K}^{\prime}$ & Winter & $19.8 \pm 1.07$ & 3.71 & F* \\
\hline & ( Soluble cations) & & Autumn & $2.00 \pm 0.16$ & & \\
\hline & $($ meq / $100 \mathrm{~g})$ & $\mathrm{K}^{+} / \mathrm{Na}^{\top}$ & Winter & $2.68 \pm 0.45$ & --- & $\mathrm{n}$ \\
\hline & & $\mathrm{Ca}^{+2}$ & Autumn & $67.7 \pm 3.02$ & $\ldots$ & $n$ \\
\hline & & & Winter & $62.0 \pm 7.02$ & --- & $\mathrm{n}$ \\
\hline & & $\mathrm{Mo}^{+2}$ & Autumn & $8.53 \pm 1.89$ & 552 & ** \\
\hline & & $\mathrm{Mg}$ & Winter & $20.5 \pm 2.88$ & 0.52 & 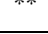 \\
\hline & & $\mathrm{Cl}^{-}$ & Autumn & $42.7 \pm 7.56$ & & \\
\hline & ( Soluble anions ) & $\mathrm{Cl}$ & Winter & $30.1 \pm 2.66$ & ---- & $\mathrm{n}$ \\
\hline & $(\mathrm{meq} / 100 \mathrm{~g})$ & $S^{-2}$ & Autumn & $6.19 \pm 0.96$ & 170 & ** \\
\hline & & $\mathrm{SO}_{4}$ & Winter & $13.2 \pm 0.46$ & 1.70 & (n) \\
\hline & & $\mathrm{P}^{+3}$ & Autumn & $16.7 \pm 0.58$ & 280 & $* *$ \\
\hline & & $P$ & Winter & $10.6 \pm 1.65$ & 2.80 & $F_{*}$ \\
\hline & Suneroxide dism & ce (SOD) & Autumn & $2.34 \pm 0.46$ & & \\
\hline & Superoxide dism & se (SUD) & Winter & $1.35 \pm 0.45$ & --- & $\mathrm{n}$ \\
\hline & Peroxidase $x$ & (DD) & Autumn & $1.52 \pm 0.51$ & 081 & $*$ \\
\hline & Peroxidase & JD) & Winter & $0.64 \pm 0.05$ & 0.81 & \\
\hline & & & Autumn & $1.42 \pm 0.09$ & & \\
\hline Antioxidant enzymes & Catalase ( & (T) ) & Winter & $0.71 \pm 0.08$ & 0.19 & $* *$ \\
\hline$\left(\mathrm{U} \mathrm{g}^{-1} \mathrm{~F} . \mathrm{wt}\right)$ & & & Autumn & $0.04 \pm 0.01$ & & $* *$ \\
\hline & Ascorbate peroxi & se (APX) & Winter & $0.15 \pm 0.01$ & 0.02 & $* *$ \\
\hline & Polyphenol oxid & $(\mathrm{PPO})$ & Autumn & $0.02 \pm 0.002$ & 0.003 & ** \\
\hline & & & Winter & $0.01 \pm 0.001$ & & \\
\hline & & & Autumn & $0.08 \pm 0.01$ & & $*$ \\
\hline & Glutathione redu & ase (GR) & Winter & $0.05 \pm 0.01$ & 0.02 & $*$ \\
\hline
\end{tabular}

LSD $=$ least significant difference, $\mathrm{n}$ : non significant., ${ }^{*}$ significant at $\mathrm{P} \leq 0.05$, ** significant at $\mathrm{P} \leq 0.01$

The eco-physiological studies in leaves

Water relation

both water content and degree of succulence showed no significant difference between autumn and winter seasons, while the osmotic potential of Asphodelus aestivus, decreased significantly during autumn (- 3.24 $\pm 0.07 \mathrm{Mpa})$ (Table 4). 
TABLE 4. Seasonal variations of water content (\%), degree of succulence (Fresh wt / dry wt.) and osmotic potential (Mpa) content of Asphodelus aestivus leaves.

\begin{tabular}{|c|c|c|c|c|}
\hline Parameters & Seasons & $\begin{array}{l}\text { Leaves } \\
\text { Mean } \pm\end{array}$ & LSD & Sig. \\
\hline \multirow{2}{*}{ Water content $(\%)$} & Autumn & $88.2 \pm 1.94$ & \multirow{2}{*}{$\mathrm{n}$} & \multirow{2}{*}{---} \\
\hline & Winter & $88.0 \pm 0.72$ & & \\
\hline \multirow{2}{*}{$\begin{array}{l}\text { Degree of succulence } \\
\text { (fresh wt / dry wt ) }\end{array}$} & Autumn & $8.65 \pm 1.37$ & \multirow{2}{*}{$\mathrm{n}$} & \multirow{2}{*}{---} \\
\hline & Winter & $8.37 \pm 0.51$ & & \\
\hline \multirow{2}{*}{ Osmotic potential (Mpa) } & Autumn & $-3.24 \pm 0.07$ & \multirow{2}{*}{0.11} & \multirow{2}{*}{$* *$} \\
\hline & Winter & $-2.39 \pm 0.02$ & & \\
\hline
\end{tabular}

LSD = least significant difference, $\mathrm{n}$ : non significant., ${ }^{*}$ significant at $\mathrm{P} \leq 0.05$, significant at $\mathrm{P} \leq 0.01$.

\section{Photosynthetic pigments}

Table 5 shows that the concentrations of pigment fractions reached the highest values during autumn. Chl (a) recorded the highest value $(1.10 \pm 0.07 \mathrm{mg} /$ g F.wt ) followed by carotenoids $(0.83 \pm 0.03 \mathrm{mg} / \mathrm{g}$ F.wt $)$ then $\mathrm{Chl}$ (b) $(0.47 \pm 0.02 \mathrm{mg} / \mathrm{g}$ F.wt $)$. Such increase in Chl (b), $(\mathrm{a}+\mathrm{b})$, carotenoids and total pigments was highly significant in autumn, whereas, no significant differences were observed for $\mathrm{Chl}(\mathrm{a})$ and $(\mathrm{a} / \mathrm{b})$ ratio.

\section{Carbohydrate content}

the obtained results clearly showed significant differences between both seasons for all carbohydrate fractions. On the contrary, the highest value of soluble sugars was recorded during winter $(6.25 \pm 0.55 \mathrm{~g} \%)$ (Table 5).

\section{Lipid content}

the difference in contents of total lipids and phospholipids were found to be significant in both seasons (Table 5). Generally, total lipids tend to increase from $3.90 \pm 0.30$ during autumn to $14.1 \pm 3.45 \mathrm{~g} / 100 \mathrm{~g}$ dry wt. during winter while, phospholipids increased significantly during autumn $(0.91 \pm 0.06 \mathrm{~g} / 100 \mathrm{~g})$.

\section{Mineral concentrations}

it is observed from Table 6 that seasons had a significant effect on mineral concentrations except for $\mathrm{Na}^{+}, \mathrm{Mg}^{+2}, \mathrm{SO}_{4}^{-2}$. Meanwhile, results of $\mathrm{K}^{+}, \mathrm{K}^{+} / \mathrm{Na}^{+}, \mathrm{Cl}^{-}$, and $\mathrm{P}^{+3}$ showed higher values $(191.45 \pm 7.83,15.88 \pm 1.67,63.07 \pm 3.80$ and $33.12 \pm 2.99 \mathrm{meq} / 100 \mathrm{~g}$ respectively) during autumn season compared with those recorded during winter. There is a general trend for potassium accumulation to increase during autumn, while calcium concentrations tend to increase during winter. 
TABLE 5. Seasonal variations of pigments ( $\mathrm{mg} / \mathrm{g} \mathrm{F.wt}$ ), sugars ( $\mathrm{g} \%$ ), total lipids and phospholipids ( $\mathrm{g} / 100 \mathrm{~g}$ ) content of Asphodelus aestivus leaves.

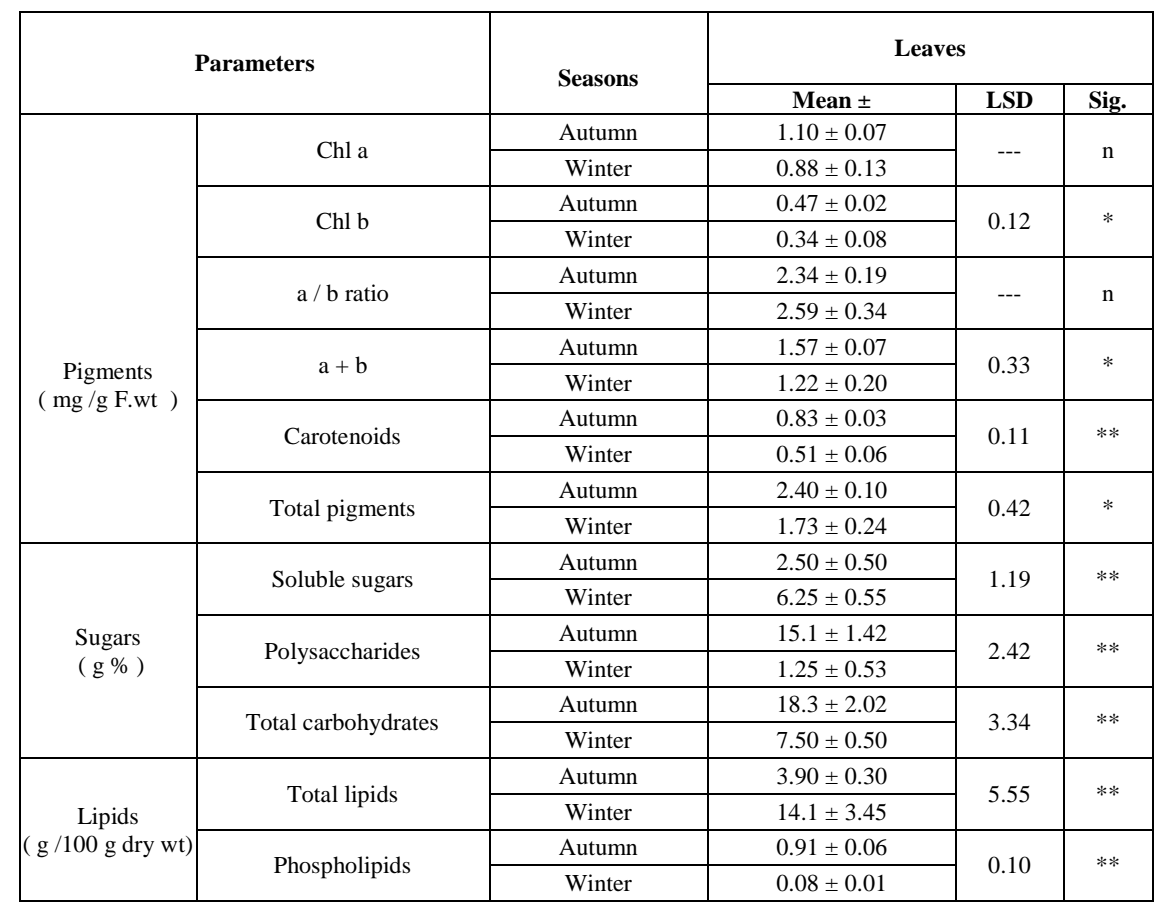

$\mathrm{LSD}=$ least significant difference, $\mathrm{n}$ : non significant., * significant at $\mathrm{P} \leq 0.05$, ** significant at $\mathrm{P} \leq 0.01$.

TABLE 6. Seasonal variations of mineral (meq / $100 \mathrm{~g}$ ) contents of Asphodelus aestivus leaves.

\begin{tabular}{|c|c|c|c|c|c|}
\hline \multirow{2}{*}{\multicolumn{2}{|c|}{ Minerals }} & \multirow{3}{*}{\begin{tabular}{|l} 
easons \\
Autumn \\
\end{tabular}} & \multicolumn{3}{|c|}{ Leaves } \\
\hline & & & \multirow{2}{*}{$\begin{array}{c}\text { Mean } \pm \\
12.17 \pm 1.74\end{array}$} & \multirow{3}{*}{ LSD } & \multirow{3}{*}{$\begin{array}{c}\text { Sig } \\
\mathrm{n}\end{array}$} \\
\hline \multirow{10}{*}{$\begin{array}{c}\text { ( Soluble cations) } \\
(\mathrm{meq} / 100 \mathrm{~g})\end{array}$} & \multirow{2}{*}{$\mathrm{Na}^{+}$} & & & & \\
\hline & & Winter & $11.28 \pm 1.48$ & & \\
\hline & \multirow{2}{*}{$\mathrm{K}^{+}$} & Autumn & $191.5 \pm 7.83$ & \multirow{2}{*}{27.7} & \multirow{2}{*}{$* *$} \\
\hline & & Winter & $61.54 \pm 15.4$ & & \\
\hline & \multirow{2}{*}{$\mathrm{K}^{+} / \mathrm{Na}^{+}$} & Autumn & $15.88 \pm 1.67$ & \multirow{2}{*}{2.97} & \multirow{2}{*}{$* *$} \\
\hline & & Winter & $5.41 \pm 0.80$ & & \\
\hline & \multirow{2}{*}{$\mathrm{Ca}^{+2}$} & Autumn & $59.73 \pm 4.46$ & \multirow{2}{*}{16.2} & \multirow{2}{*}{$*$} \\
\hline & & Winter & $79.20 \pm 9.07$ & & \\
\hline & \multirow{2}{*}{$\mathrm{Mg}^{+2}$} & Autumn & $9.80 \pm 3.40$ & \multirow{2}{*}{---- } & \multirow{2}{*}{$\mathrm{n}$} \\
\hline & & Winter & $9.40 \pm 1.80$ & & \\
\hline \multirow{6}{*}{$\begin{array}{c}\text { ( Soluble anions ) } \\
(\mathrm{meq} / 100 \mathrm{~g})\end{array}$} & \multirow{2}{*}{$\mathrm{Cl}^{-}$} & Autumn & $63.07 \pm 3.80$ & \multirow{2}{*}{7.24} & \multirow{2}{*}{$* *$} \\
\hline & & Winter & $45.20 \pm 2.43$ & & \\
\hline & \multirow{2}{*}{$\mathrm{SO}_{4}^{-2}$} & Autumn & $7.52 \pm 1.30$ & \multirow{2}{*}{---- } & \multirow{2}{*}{$\mathrm{n}$} \\
\hline & & Winter & $8.97 \pm 0.20$ & & \\
\hline & \multirow{2}{*}{$\mathrm{P}^{+3}$} & Autumn & $33.12 \pm 2.99$ & \multirow{2}{*}{6.52} & \multirow{2}{*}{$* *$} \\
\hline & & Winter & $18.86 \pm 2.75$ & & \\
\hline
\end{tabular}

LSD $=$ least significant difference, $\mathrm{n}$ : non significant., * significant at $\mathrm{P} \leq 0.05, * *$ significant at $\mathrm{P} \leq 0.01$. 
Antioxidant enzymes

Table 7 shows that the activities of SOD, GR and GPX increased $(2.59 \pm 0.24$, $0.14 \pm 0.01,0.84 \pm 0.04 \mathrm{U} / \mathrm{g}$ F.wt , respectively) during autumn season. In contrast, a high increase of POX $(0.78 \pm \mathrm{U} / \mathrm{g}$ F.wt) was observed in winter while, no significant differences were observed for PPO, CAT or APX.

TABLE 7. Seasonal variations of antioxidant enzymes $\left(\mathrm{Ug}^{-1} \mathbf{F}\right.$.wt) activity of Asphodelus aestivus leaves.

\begin{tabular}{|c|c|c|c|c|c|}
\hline & \multirow{2}{*}{ Enzymes } & \multirow{2}{*}{ Seasons } & \multicolumn{3}{|c|}{ Leaves } \\
\hline & & & Mean \pm & LSD & Sig. \\
\hline \multirow{14}{*}{$\begin{array}{c}\text { Antioxidant } \\
\text { enzymes } \\
\left(\mathrm{Ug}^{-1} \mathrm{~F} . \mathrm{wt}\right)\end{array}$} & \multirow{2}{*}{$\begin{array}{c}\text { Superoxide dismutase } \\
\text { (SOD) }\end{array}$} & Autumn & $2.59 \pm 0.24$ & \multirow{2}{*}{0.52} & \multirow{2}{*}{$* *$} \\
\hline & & Winter & $1.58 \pm 0.23$ & & \\
\hline & \multirow{2}{*}{ Peroxidase ( POD ) } & Autumn & $0.25 \pm 0.05$ & \multirow{2}{*}{0.08} & \multirow{2}{*}{$* *$} \\
\hline & & Winter & $0.78 \pm 0.01$ & & \\
\hline & \multirow{2}{*}{ Catalase ( CAT ) } & Autumn & $0.59 \pm 0.09$ & \multirow{2}{*}{---- } & \multirow{2}{*}{$\mathrm{n}$} \\
\hline & & Winter & $0.72 \pm 0.08$ & & \\
\hline & \multirow{2}{*}{ Ascorbate peroxidase (APX) } & Autumn & $0.13 \pm 0.01$ & \multirow{2}{*}{----} & \multirow{2}{*}{$\mathrm{n}$} \\
\hline & & Winter & $0.12 \pm 0.05$ & & \\
\hline & \multirow{2}{*}{ Polyphenol oxidase ( $\mathrm{PPO}$ ) } & Autumn & $0.03 \pm 0.01$ & \multirow{2}{*}{---- } & \multirow{2}{*}{$\mathrm{n}$} \\
\hline & & Winter & $0.01 \pm 0.001$ & & \\
\hline & \multirow{2}{*}{ Glutathione reductase (GR) } & Autumn & $0.14 \pm 0.01$ & \multirow{2}{*}{0.02} & \multirow{2}{*}{$*$} \\
\hline & & Winter & $0.11 \pm 0.01$ & & \\
\hline & \multirow{2}{*}{$\begin{array}{c}\text { Glutathione peroxidase ( } \\
\text { GPX ) }\end{array}$} & Autumn & $0.84 \pm 0.04$ & \multirow{2}{*}{0.09} & \multirow{2}{*}{$* *$} \\
\hline & & Winter & $0.20 \pm 0.04$ & & \\
\hline
\end{tabular}

\section{Discussion}

One of the prominent features of Mediterranean climate is its periodicity, to which $A$ aestivus has responded by synchronizing the annual development of Its biological cycle. Changes in water and energetic status are associated with the particular phenophases and serve a double purpose to the species, i.e., to face climatic stresses and satisfy growth and reproduction needs. The parallel examination of the above ground and the underground structures reveals the contribution of each of these two plant parts to the overall response of the species in this fluctuating environment (Rhizopoulou et al., 1997).

Soil moisture content tended to decrease generally during autumn while, the electrical conductivity was very low. Soil reaction was weakly alkaline. Calcium was the dominant cation in the soil followed by $\mathrm{Mg}^{+2}$.Chlorides were generally the dominant anions followed by bicarbonate. Results also revealed that the surface layer achieved the highest amount of total cations. Total anions differed slightly by seasons and depths. The soil moisture content in the upper depth was lower than that of lower one which might be attributed to the fact that surface layers of the desert soil is subjected to evaporation. The other most important edaphic factor that affects moisture availability and subsequently the distribution of plant was soil texture (Wang et al., 2011). 
Soil was sandy loam formed mainly of medium and fine sands. So, soil supports the growth of A. microcarpus, especially the growing of mild deeper root of the plant .It is possible to utilize up to $75 \%$ of the available soil water without having a deleterious impact on plant development (Wang et al., 2011). Migahid et al., (1974) studied the most sands of Sedi Barrani region, and found that the penetrability was greatest in the soil surface and it decreases appreciably below certain depths. Such increased resistance in depth may be due to either the formation of a carbonate accumulation zone or to increased proportion of fine soil. This conclusion has been justified by the results of granulometric analysis and calcium carbonate content obtained from the present study. Granulometric analysis showed that fine and medium sand were increased in the upper layer, and then decreased while calcium carbonate was found in the lower layer during autumn.

A decrease in water content and succulence in root tubers were noticed during autumn. This may be due to growing buds and leaves which are large recipients of water during the storage period (dormancy) under drought stress (Kamenetsky and Okubo, 2013).Water content in tubers, which has been transferred to the buds can play an important role in the complete development of flowers in winter. These values were in accordance with the climatic conditions of the study area, which is characterized as a Mediterranean climate (Kirmizi et al., 2014).

A decrease in sugars at the beginning of autumn was recorded. Sucrosecleaving enzymes (invertases and sucrose-synthase) can be activated when there is a demand for metabolic carbohydrate and energy (Kamenetsky and Okubo, 2013). Soluble sugars might serve as osmolytes during water stress. The increase of minerals except for $\mathrm{Mg}^{+2}$ and sulfates, during autumn suggested that geophytes can overcome the unfavorable growing conditions by persisting in the form of a subterranean organ that can accumulate sufficient nutrients available for the fast development of the above-ground organs during annually cycling periods of suitable conditions. The increase of magnesium in tuber during winter in the present study, maybe ascribed to increase root growth and root surface area which may be involved in increasing the uptake of water and nutrients (Richardson et al., 2002).The stored reserves can allow geophytes to develop successfully even at very low initial external energy supply. Moreover, the antioxidant system in plants can act as a protective mechanism that confers tolerance to environmental stresses.

A higher amount of ROS triggers up the regulation of the activity of antioxidant enzymes, which in turn protects plants from oxidative stress. This explains the increase of the antioxidant enzymes except for APX during autumn. It is known that APX and CAT belong to two different classes of $\mathrm{H}_{2} \mathrm{O}_{2}$ scavenging enzymes where APX is responsible for the modulation of stress, whereas CAT is responsible for the removal of excess $\mathrm{H}_{2} \mathrm{O}_{2}$ during stress. (Chen et al., 2010). 
Water content and succulence showed no significant increase in autumn, which may be helpful to support their physio-biochemical processes efficiently under water stress conditions. Under adverse environmental conditions, plant close their stomata to inhibit rapid water loss (Ozfiden et al., 2013). The decline in osmotic potential was significantly affected by seasons due to osmotic adjustment. Osmotic adjustment may allow growth to continue at low water potential (Akinci and Lösel, 2012).

Photosynthetic pigments as well as pigment fractions in Asphodelus aestivus plant in the current study reached the highest values in autumn. Chlorophyll content can act as an indicator of nutrient status (Richardson et al., 2002). Carotenoids have essential functions in photosynthesis and photo protection, besides their structural roles, they are well known for their antioxidant activity by quenching the singlet oxygen, inhibiting lipid peroxidation and stabilizing membranes. They also play a critical role in the assembly of the light-harvesting complex and in the dissipation of excess energy.

Data of the present study clearly demonstrate that soluble sugar and other carbohydrates in leaves were altered, which could be a metabolic signal in response to drought (Akinci and Lösel, 2012). During active aboveground growth (late winter), carbohydrates accumulated due to photosynthesis have been transported towards the underground organs, resulting in bulb enlargement. After a critical bulb size was reached, the flower meristem was induced and differentiated at the end of summer. Dormancy was broken in autumn, resulting in shoot and flower bud growth that continued throughout the winter. For most of the year, however, these plants can undergo an extended non-photosynthetic growth period (Khodorova and Boitel-Conti, 2013). Total lipids were decreased in both of tubers and leaves in autumn but phospholipids were increased in leaves. During exposure to stress (tubers during dormancy in the present study), the major role of phospholipids as backbones of cellular membranes can serve as precursors for the generation of second-messenger molecules.

As a result, phospholipids could substitute the lack of glycolipids or galactolipid in extraplastidial membranes which necessary for the stability of the chloroplast membrane since absorption by the root stops (tubers of the studied plant), phosphate may increase leading to an increase in phospholipid biosynthesis, in addition to overall lipids degradation. The uptake of $\mathrm{P}^{+3}$ by tubers may increase the level of phospholipids in autumn (next seasons to dormancy) in the present study and transfer it to the growing leaves in the same period. Generally, geophytes are highly flexible to adverse seasons (summer dormancy under drought stress), as sufficient resources are carried over to the next season to allow a population to survive.

Results of $\mathrm{K}^{+}, \mathrm{Na}^{+}$and $\mathrm{K}^{+} / \mathrm{Na}^{+}$ratio, recorded the highest values during dry season (autumn).This may be due to the presence of common proteins that transport $\mathrm{Na}^{+}$and $\mathrm{K}^{+}\left(\mathrm{Na}^{+} / \mathrm{K}^{+}\right.$symporters $), \mathrm{Na}^{+}$competes with $\mathrm{K}^{+}$for intracellular influx. Thus, external $\mathrm{Na}^{+}$negatively impacts intracellular $\mathrm{K}^{+}$influx. Most cells

Egypt. J. Bot., Vol. 56, No. 1 (2016) 
maintain relatively high $\mathrm{K}^{+}$with low concentrations of $\mathrm{Na}^{+}$in the cytosol. Increases in $\mathrm{K}^{+}$demand maintain the photosynthesis and protect chloroplasts from oxidative damage, this in turn could enhance photosynthetic rate, plant growth and maintenance of a high $\mathrm{pH}$ in stroma against the photo-oxidative damage (Waraich et al., 2011). In general, $\mathrm{Ca}^{+2}$ channels are not entirely $\mathrm{Ca}^{+2}$ selective but also show permeability to other cations. However, the genes encoding plasma membrane $\mathrm{Ca}^{+2}$ channels remain less well-clarified (Vahdati and Lotfi, 2013).

Calcium ions act as a second messenger in intracellular signal transduction during Absicic acids (ABA) signaling. Kirnak et al. (2003) stated that water stress could cause $\mathrm{Ca}^{+2}$ reduction and suggested antagonistic effects of $\mathrm{Ca}^{+2}$ uptake on potassium. Data showed an increase of $\mathrm{P}^{+3}$ in the studied plant during dry season which is supported by the fact that $\mathrm{P}^{+3}$ is required for processes including the storage and transfer of energy, photosynthesis, the regulation of some enzymes, and the transport of carbohydrates. Phosphorus also maintains the cell turgidity by maintaining the high leaf water potential, which in turn increases the stomatal conductance and increases the photosynthetic rate, higher cell membrane stability, water relations and drought tolerance.

Increase of chloride anion(during autumn) may be due to the signaling cascade mediated by ABA causing the efflux of $\mathrm{K}^{+}$and $\mathrm{Cl}^{-}$and the removal of organic solutes from guard cells, thereby reducing cellular turgor and leading to rapid stomatal closure to prevent water loss by transpiration. Chloride anions shared in membrane depolarization activation, so membrane depolarization activates outward $\mathrm{K}^{+}$channels and facilitates the efflux of organic solutes (Song and Matsuoka, 2009). Data of the present study showed that the activities of SOD, GR and GPX in leaves increased, while POD decreased during dry season. However, changes in activities of CAT and APX were not significant (slight increase in APX and decrease in CAT).

The APX and GPX are up-regulated when CAT activity is reduced in plants (Sofo et al., 2015). The SOD is one of the most important detoxifying enzymes which is responsible for the scavenging of toxic $\mathrm{O}^{-2}$ in different cell organelles. SOD catalyzes the disproportion of $\mathrm{O}_{2}{ }^{*}$ into $\mathrm{H}_{2} \mathrm{O}_{2}$ and $\mathrm{O}_{2}$ susceptible to oxidative stress (Chen et al., 2010). On the other hand, GR catalyzes the NADPH-dependent formation of a disulphide bond in glutathione disulphide (GSSG) which is important for maintaining the reduced pool of GSH. The role of GSH and GR in $\mathrm{H}_{2} \mathrm{O}_{2}$ scavenging has been well established in the HalliwellAsada pathway.

In parallel, glutathione peroxidases (GPX) reduce $\mathrm{H}_{2} \mathrm{O}_{2}$ and organic hydroperoxides by ascorbate-independent thiol-mediated pathways (Iqbal et al., 2006). GPXs may play a role in other parts of antioxidant metabolism, including the removal of lipid peroxides. GPXs generally increase in plants subjected to environmental constraints such as water deficit (Navrot et al., 2006) therefore, the activity of antioxidant enzymes increased to scavenge the increase in ROS

Egypt. J. Bot., Vol. 56, No. 1 (2016) 
and protect macromolecules in cells as an activated defense mechanism (Sofo et al., 2015) and to suggest the tolerance capacity of A. aestivus plant from oxidative damage.

\section{Conclusion}

Asphodelus aestivus Brot. has developed different defense strategies against drought and oxidative stresses in its habitat during life form. The two plant parts respond differently to stress associated with the major phenophases of the species. A. aestivus achieves "palliation" by an increase of specific elements and antioxidant enzymes in tubers and growing leaves during active life cycle. Palliation means that the impact of the stress factor is mitigated and cause physiological changes. This geophyte has undergone adaptations and development of subterranean organs that contain specialized storage compounds in order to survive extreme environments. A. aestivus may have evolved a kind of adaptation, it may can maintain the tissues (physiological integrity) during dehydration (low soil moisture during autumn) and upon rehydration (winter), and able to repair any damage that has been caused by ROS. This was proposed for the aboveground vegetative tissues and the storage root tubers.

\section{References}

A.O.A.C (2000) "Official Methods of Analysis", $17^{\text {th }}$ ed. Association of Official Analytical Chemists .Washington D.C.,U.S.A.

Akinci, S. and Lösel, D. M. (2012) "Plant Water Stress Response Mechanisms": I. Md. Rahman M, Hasegawa, H. (Eds).Water Stress .Rijeka, Croatia. In Tech15-42.

Boulos , L. (2005) "Flora of Egypt" Ed. Al - Hadara Publishing ,Cairo , Egypt.

Chaplin, M. F. and Kennedy, J. F. (1994) Carbohydrate analysis. A practical approach, 2 (1-4): 76- 77.

Chapman, H. D. and Pratt, P. F. (1961) "Methods of Analysis for Soils, Plants and Water". University of California, U.S.A, Pp. 309.

Chen, Q., Zhang, M. and Shen, S. (2010) Effect of salt on malondialdehyde and antioxidant enzymes in seedling roots of Jerusalem artichoke (Helianthus tuberosus L.). Acta Physiol Plant, 33:273-278.

Connerty, H. V., Briggs, A. R. and Eaton, E. H. J. (1961) Clin.Chem., 7: 37-580.

Foyer, C. H. and Halliwell, B. (1976) The presence of glutathione and glutathione reductase in chloroplasts: a proposed role in ascorbic acid metabolism. Planta, 133: 21-25.

Goel, A., Goel, A. K. and Sheoran, I. S. (2003) Changes in oxidative stress enzymes during artificial agein in cotton (GossypiumhirsutumL.) seeds. J. Plant. Physiol., 160: 1093-1100.

Egypt. J. Bot., Vol. 56, No. 1 (2016) 
Homme, P. M., Conalez, B. and Billard, J. (1992) Carbohydrate content, fructose and sucrose, enzyme activities in roots, stubble and leaves of rye grass (Lolium perenne L.) as affected by source / sink modification after cutting. J. Plant Physiol., 140: 282-291.

Hammerschmidt, R., Nuckles, E. M. and Kúc, J. (1982) Association of enhanced peroxidase activity with induced systemic resistance of cucumber to Colletotrichum lagenarium. Physiol. Plant Pathol., 20: 73-82.

Harris, D. C. (1998) "Quantitative Chemical Analysis". Hand -Book, W.H. Freeman and Company, California, New York. U.S.A.

Hoolbrook, N. M. and Putz, F. E. (1996) From Epiphyte to tree: differences transition in growth forms in eight species of hemiepiphytes. Plant. Cell and Environ., 19: 631-642.

Iqbal, A., Yabuta, Y., Takeda, T., Nakano, Y. and Shigeoka, S. (2006) Hydroperoxide reduction by thioredoxin-specific glutathione peroxidase isoenzymes of Arabidopsis thaliana. FEBS J., 273: 5589-5597.

Jackson, M. L. (1967) "Soil Chemical Analysis". Prentice - Hall of India Private, New Delhi, India.

Jackson, W. A. and Thomas, G. W. (1960) Effect of $\mathrm{KCl}$ and dolomite limestone on growth and ion uptake of Sweet Potato. Soil. Sci., 89:347-352.

James, C.S. (1995) "Analytical Chemistry of Foods" . Blackle Academic and Professional Publisher. An imprint of Champmen and Hall, 178pp.

Kamenetsky, R. and Okubo, H. (2013) "Ornamental Geophytes: From Basic Science to Sustainable Production". CRC Press: Boca Raton F L, USA. Pp. 578.

Khodorova, N. V. and Boitel-Conti, M. (2013) The role of temperature in the growth and flowering of geophytes. Plants, 2: 699-711.

Kirmizi, S., Güleryüz, G. and Arslan, H. (2014) Protein mobilisation and proteolytic activities in root tubers of Asphodelus aestivus. Turk. J. Bot., 38: 302-308.

Kirnak, H., Kaya, C., Higgs, D. and Tas, I. (2003) Responses of drip irrigated bell pepper to water stress and different nitrogen levels with or without mulch cover. $J$. Plant Nutr., 26: 263-277.

Mayer, A. M. , Harel, E. and Shaul, R. B. (1965) Assay of catechol oxidase a critical comparison of methods. Phytochem, 5:783-789.

Metzner, H., Rau, H. and Senger, H. (1965) Unter suchung enzursyn chronisier barke it einzelner- pigment. "Mangol Mutanten Von Chloella". Planta, Pp. 65, 186.

Migahid, A., El -Sharkawi, H.M., Batanouny, K.H. and Shalaby, A.F. (1974) Phytosociological and ecological studies of maktila sector of sidi-barrani I. sociology of the communities. Feddes Repertorium Berlin., 15 (1):747-760.

Misra, M. and Fridovich, F. (1972) The role of superoxide anion in the autoxidation of epinephrine and a simple assay for superoxide dismutase. Journal of Biological Chemistry, 247 (10): 3170-3175.

Egypt. J. Bot., Vol. 56, No. 1 (2016) 
Nakano, Y. and Asada, K. (1981) Hydrogen peroxide is scavenged by ascorbate specific peroxides in spinach chloroplasts, Plant Cell Physiol., 22: 867-880.

Navrot, N., Collin, V., Gualberto, J., Gelhaye, E., Hirasawa, M., Rey, P., Knaff, D. B., Issakidis, E., Jacquot, J. P. and Rouhier, N. (2006) Plant glutathione peroxidases are functional peroxiredoxins distributed in several subcellular compartments and regulated during biotic and abiotic stresses. Plant Physiol., 142: 1364-1379.

Ozfidan, C., Turkan, I., Sekmen, A.H. and Seckin, B. (2013) Time course analysis of $\mathrm{ABA}$ and non-ionic osmotic stress-induced changes in water status, chlorophyll fluorescence and osmotic adjustment in Arabidopsis thaliana wild-type (Columbia) and ABA-deficient mutant $\left(\mathrm{ABA}_{2}\right)$. Environmental and Experimental Botany, 86: 44 - 51.

Richardson, A.D., Duigan, S.P. and Berlyn, G.P. (2002) An evaluation of non invisible methods to estimate foliar chlorophyll content. New Physiologist, 153(1): 185-194.

Rhizopoulou, S., Pantis, J. D., Triantafylli, E. and Vokou, D. (1997) Ecophysiological adaptations of Asphodelus aestivus to Mediterranean climate periodicity: water relations and energetic status. Ecography. Copenhagen. 20: 626-633.

Rowell, D. L. (1994) "Soil Science Methods and Applications". Longman Publishers, Singapors, Pp. 53, 229.

Simmelsgaard, S. E. (1976) Adaptation to water stress in wheat. Physiol. Plant, 37:167-174.

Shewry, P. R. (2003) Tuber Storage Proteins . Annals of Botany, 91(7):755-769.

Smith, D., Poulsen, G.M. and Raguse, C.A. (1964) Extraction of total available carbohydrates from grass and legume tissues. Plant Physiol., 39: 960-969.

Sofo, A., Scopa, A., Nuzzaci, M. and Vitti, A. (2015) Ascorbate peroxidase and catalase activities and their genetic regulation in plants subjected to drought and salinity stresses. Int. J. Mol. Sci., 16: 13561-13578.

Song, X. and Matsuoka, M. (2009) Bar the windows: an optimized strategy to survive drought and salt adversities. Genes \& Development, 23:1709-1713.

Vahdati, K. and Lotfi, N. (2013) Abiotic stress tolerance in plants with emphasizing on drought and salinity stresses in Walnut. licensee In Tech., 10: 307-365.

Wang, Y., Ma, F., Li, M., Liang, D. and Zou, J. (2011) Physiological responses of kiwifruit plants to exogenous ABA under drought conditions. Plant Growth Regul, 64: 63-74.

Waraich, E. A., Ahmad, R., Saifullah, A., Ashraf, M. and Ehsanullah, Y. (2011) Role of mineral nutrition in alleviation of drought stress in plants. AJCS, 5(6): 764-777.

Wendel, A. (1981) Glutathione peroxidase. Methods enzymol., 77: 325 - 333. 


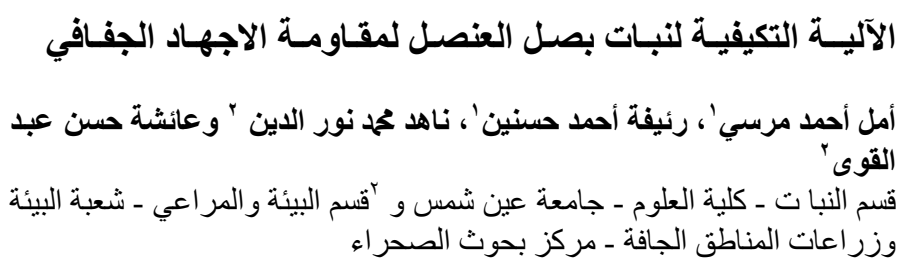

- يهدف البحث لدراسة الاستجابات الفسيولوجية الناجمة عن تأثير الاجهاد التأكسدي

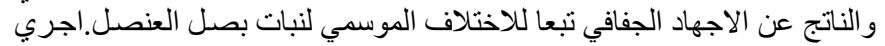

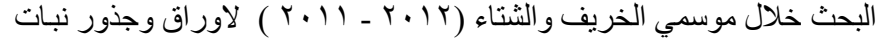

بصل العنصل في منطقة سيدي بر اني.كمـا اجريت النحاليل الفيزيائية و الكيميائية

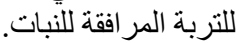

ـ تم دراسـة المحتوي المسائي والعصـاري للاور راق و الجذور والاجهاد الاسموزي

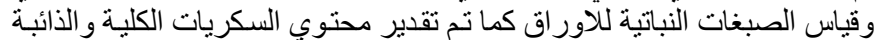

والمتعددة بالاضـافة الي الدهون الكلية والفسفوليبيدات وتقدير الاملاح المعدنية

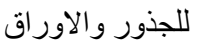

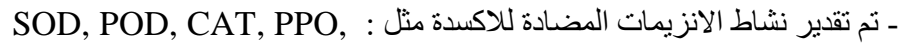

APX, GR \& GPX

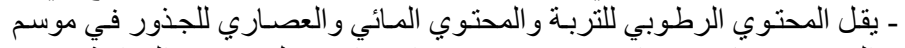

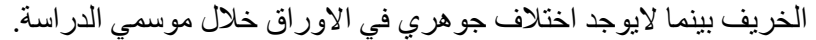

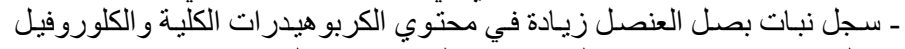

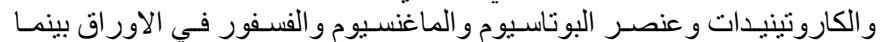

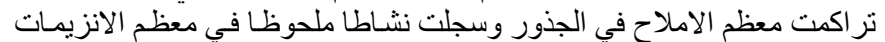

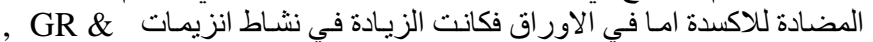

.GPX, SOD

ـ اظهرت الدراسة ان نبات بصل العنصل يتخذ استر اتيجية دفاعية خاصـة اتجاه

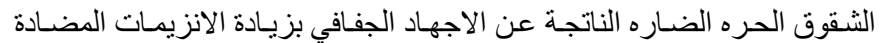

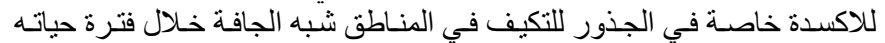

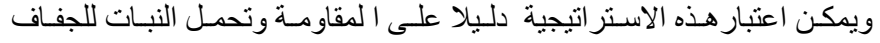

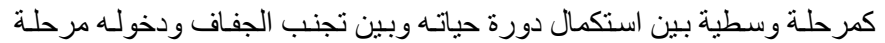

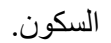

